\section{THE TRI-SERVICE ANAESTHETISTS IN TRAINING MEETING 2013}

David Evans

This year's annual meeting met at the Defence Academy at Shrivenham and attracted the vast majority of trainee anaesthetists in the military. We were fortunate to have Professor Mike P Grocott as a speaker to discuss his work on the physiology of high altitude and its similarities to the physiological stresses imposed on very unwell patients in the intensive care unit.

The wider military and medical context of deployed medical support was covered by an insightful lecture by Lt Col de Rouffignac Commanding Officer of 3 Medical Regiment who covered current deployed operations in Afghanistan and went on to give some predications for the future shape of a deployed medical force and how we were likely to work with other medical elements in the future.

Dr Liam Brennan, who is soon to take up the role of Vice President of the Royal College of Anaesthetists and has been heavily involved in the College in a variety of roles, discussed a wide range of issues related to training, post-certificate of completion of specialist training fellowships and revalidation. We were fortunate that Lt Col de Rouffignac and Dr Brennan were able to join us for a 'relaxed,' formal dinner at the Defence Academy.

The final day included formal updates from those senior officers involved in training and leading the anaesthetic cadre and the abstracts below competed for the David Hughes memorial prize presentation, which was won by Captain A Kumar.

\section{LOCAL ATTITUDES AND USE OF RESERVE GAS CYLINDERS ATTACHED TO ANAESTHETIC MACHINES: IMPLICATIONS FOR LOCAL PRACTICE}

M Creed, ${ }^{1}$ A Morgan ${ }^{2}$ Army Anaesthetic Core Trainee, West Midlands Deanery; ${ }^{2}$ Anaesthetic Core Trainee, West Midlands Deanery

Background A local audit ${ }^{1}$ prompted assessment of checks $^{2}$ on, attitudes to and use of reserve gas cylinders. Methods A survey was designed to assess local attitudes and practice. This was sent by email to all anaesthetists in our department to complete. Results Consultants, staff and associate specialists and trainees responded. The mean level of experience was 12.8 years. Respondents stated routine cylinder checking was by operating department practitioners (77\%), junior colleagues (33\%) and individual respondents (88\%). 53\% check only oxygen.96.2\% check cylinder pressure. Security of attachment, colour, capacity and expiry are checked significantly less often.11\% of respondents had experienced pipeline failure. Oxygen was considered essential by $100 \%$, nitrous oxide by $18.5 \%$ and air by $3.7 \% .100 \%$ of respondents indicated objections to removal of oxygen, $96 \%$ would not object to removal of nitrous oxide and $52 \%$ to removal of air. Discussion We found routine cylinder checks were generally short of the prescribed standard-particularly for gases other than oxygen. We infer from responses that nitrous oxide and air are less likely to be used in a main pipeline failure event.Nitrous oxide and air cylinders may warrant removal; this may offer modest cost savings. Conclusions Local education to encourage improvement in cylinder checks will occur. Open discussion and debate on the option of removing nitrous oxide and air cylinders from machines is planned to occur at the next quality improvement meeting.

\section{REFERENCES}

1 Creed M. Audit of the expiry dates of reserve gas cylinders attached to anaesthetic machines; June 2013.

2 Association of Anaesthetists of Great Britain and Ireland. Checking Anaesthetic Equipment 2012. Anaesthesia 2012;67:660-8.

\section{REWARMING IN TRAUMA AND RELATIONSHIPS WITH COAGULATION}

D Evans, D Keene Anaesthetic Trainees Role 3 Camp Bastion Hospital, Afghanistan

Introduction We know that temperature changes in trauma patients can have a variety of deleterious consequences. Hypothermia gives rise to coagulopathy, tachypnoea, vasoconstriction and shivering, while restoration of normothermia improves cardiac function and visceral blood flow. Methods Patients' temperatures were taken on admission to the Role 3; concurrently, serum lactate and the rotem parameters clotting time (CT), clot formation time (CFT) and mean clot firmness (MCF) were measured. The time to attain normothermia was recorded along with lactate, MCF, CT and CFT. We looked at relationships between initial recorded temperatures and the measured coagulation and perfusion parameters and subsequent changes in these parameters as warming took place. Results From a total of 34 patients, 30 had admission temperatures of less than $36.5^{\circ} \mathrm{C}$. The mean admission temperature was $35.2^{\circ} \mathrm{C}$. Importantly, all the patients left the operating theatre warm with a mean time from point of injury to achieving $36.5^{\circ} \mathrm{C}$ of $3 \mathrm{~h}$ and $31 \mathrm{~min}$. There appeared to be an inverse relationship between initial temperature and lactate; this was not found to be statistically significant (Figure 1). None of the other remaining measured parameters appeared to show any correlation with temperature. A two-tailed student $t$ test was used to analyse the pre-warming and post-warming comparisons. We achieved a statistically significant improvement in lactate $\mathrm{p}<0.002$ with a mean reduction of $1.36 \mathrm{mmol} / \mathrm{L}$. None of the other parameters achieved significant differences. Discussion We were successful in implementing the Role 3 environmental warming guidelines, achieving a $100 \%$ rewarming by the time the patient left the operating theatre. We know that temperature can affect clotting and coagulopathy is bad and therefore warming can help. However, there are many additional interventions occurring concurrently such as blood product transfusion which confound any further analysis.

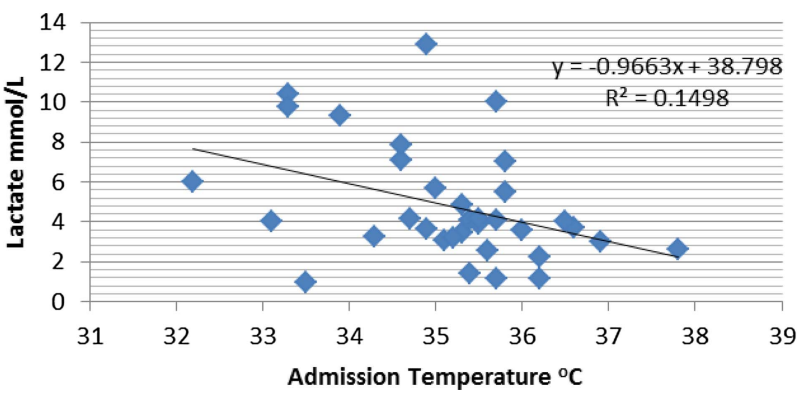

Figure 1 The relationship between initial measured temperature and serum lactate. 


\section{LARYNGOSCOPE LIGHT FAILURE: THE IMPORTANCE OF INCIDENT REPORTING}

A Kumar, C Gough Frimley Park Hospital NHS Trust, Frimley, Surrey UK

Background In December 2012, incident reporting at the monthly Anaesthetic Department risk management meeting identified a problem with the light on the Marshall Visionary Fibre Optic Laryngoscope blade failing during intubation. This was impacting on patient safety on a number of levels from simply reducing the chance of a first pass tracheal intubation to precipitating a potentially life threatening critical incident. It was also eroding the confidence of the anaesthetists in their equipment. We aimed to quantify the extent of this problem and to find a solution. Methods An audit of Mac 3 and Mac 4 blades was undertaken on a single day in 18 Theatres at Frimley Park Hospital (FPH), assessing whether the blades were fully working, flickering or failing. An online questionnaire was circulated to all members of the department. Results 16 (88\%) Mac 3 blades were found to be working, while 1 (6\%) flickered and 1 (6\%) failed. 12 (69\%) Mac 4 blades were fully working, while 5 (25\%) flickered and 1 (6\%) failed. The questionnaire had a $70 \%$ response rate with a total of 28 replies. Only six anaesthetists (23\%) had not experienced a flickering blade in the preceding month, while $15(53 \%)$ had experienced at least one failure. 11 (39\%) respondents had been forced to change blade during an intubation at least once in the preceding month. The problem was investigated by Marshall, and identified as a failure of the light unit. Using computer assisted design, new components were modelled and tested beyond normal tolerances. New light units were fitted to all laryngoscopes in theatres at FPH and then were re-audited. All Mac 3 and 4 blades were found to be fully working. Discussion Through incident reporting and a formal risk management process, we identified a serious problem affecting patient safety. With evidence obtained through audit, we were able to influence a major medical equipment company to modify the design and manufacture of one of its products. Marshall have now updated their entire product range leading to improved patient safety in the anaesthetic environment.

\section{THE ACUTE SURGICAL PATIENT: IMPROVING PATIENT PATHWAYS AND EXPERIENCE}

R Mellor Portsmouth Hospital Trust, Portsmouth, UK

Patients admitted with an acute abdomen experience a myriad of emotional stresses in addition to the physiological stresses of their clinical condition. This aspect of their care is often neglected and is crucial in patient reported outcome measures (PROMs); this is increasingly important in view of the Keogh report. We first performed this analysis of the patient pathway in 2010 and revisited it again in 2012, interviewing 50 patients who underwent emergency surgery in a large district general hospital. Pre-operative pain, adequacy of analgesia, type of analgesia and post-operative pain were examined, with PROMs covering explanations from anaesthetists and surgeons, privacy of conversations, ability to sleep at night and accessibility to water. These non-clinical aspects are increasingly important in the delivery of holistic patient care. The clinically related findings over the time period were remarkably similar despite inclusion of a wider variety of confidential enquiry into patient outcome and death patients. Post-operative pain remained severe in $22 \%$ of patients. However, there was an improvement in PROM results suggesting that previous interventions had had an effect with the exception of a $23 \%$ rise in patients unable to sleep at night. These findings have been fed back to the medical and nursing directors with an aspiration to continually improve patient care further; more detailed assessments of the emergency patient's journey are planned for the future to ensure continued delivery of high quality encompassing care for all.

Provenance and peer review Not commissioned; internally peer reviewed.

Correspondence to Sqn Ldr David Evans, 18 Montagu Road, Brampton, Huntingdon, Cambridgeshire PE28 4QG, UK; daviddoctordave@aol.com 